Part 1: clinical and pathophysiologic considerations. Obstet. Gynecol. Surv. 57:598-618.

3. Podjarny, E., Baylis, C., and Losonczy, G. 1999. Animal models of preeclampsia. Semin. Perinatol. 23:2-13.

4. Maynard, S.E., et al. 2003. Excess placental soluble fms-like tyrosine kinase 1 (sFlt1) may contribute to endothelial dysfunction, hypertension, and proteinuria in preeclampsia. J. Clin. Invest. 111:649-658. doi:10.1172/JCI200317189.
5. Eremina, V., et al. 2003. Glomerular-specific alter ations of VEGF-A expression lead to distinct congenital and acquired renal diseases. J. Clin. Invest. 111:707-716. doi:10.1172/JCI200317423.

6. King, B.F. 1987. Ultrastructural differentiation of stromal and vascular components in early macaque placental villi. Am. J. Anat. 178:30-44

7. Goldman-Wohl, D., and Yagel, S. 2002. Regulation of trophoblast invasion: from normal implantation to pre-eclampsia. Mol. Cell. Endocrinol. 187:233-238.
8. Zhou, Y., et al. 2002. Vascular endothelial growth factor ligands and receptors that regulate human cytotrophoblast survival are dysregulated in severe preeclampsia and hemolysis, elevated liver enzymes, and low platelets syndrome. Am. J. Pathol. 160:1405-1423.

9. Mattot, V., et al. 2002. Loss of the VEGF(164) and VEGF(188) isoforms impairs postnatal glomerular angiogenesis and renal arteriogenesis in mice. J. Am. Soc. Nephrol. 13:1548-1560.

\title{
Nephrolithiasis: site of the initial solid phase
}

\author{
David A. Bushinsky
}

University of Rochester School of Medicine and Dentistry and the Nephrology Unit, Strong Memorial Hospital, Rochester, New York, USA

J. Clin. Invest. 111:602-605 (2003). doi:10.1172/JCI200318016.

Most cases of nephrolithiasis are associated with the relatively common metabolic abnormality of idiopathic hypercalciuria (1). These patients generally absorb an excess amount of dietary calcium leading to increased urine calcium excretion and supersaturation with respect to calcium oxalate and calcium phosphate; they subsequently form stones. Other patients with nephrolithiasis, who have had an intestinal bypass procedure, absorb oxalate in excess leading to increased urine oxalate excretion and supersaturation with respect to calcium oxalate; they also subsequently form stones. In these and other causes of nephrolithiasis, the site of the initial solid phase has long been the subject of debate. Over 65 years ago, A. Randall demonstrated that interstitial crystals located at, or adjacent to, the papillary tip, Randall's plaques, were common in

\footnotetext{
Address correspondence to: David A. Bushinsky, University of Rochester School of Medicine and Dentistry, Nephrology Unit, Strong Memorial Hospital, 601 Elmwood Avenue, Box 675, Rochester, New York 14642, USA. Phone: (585) 275-3660;

Fax: (585) 442-9201; E-mail:

David_Bushinsky@urmc.rochester.edu.

Conflict of interest: The author has declared that no conflict of interest exists.
}

stone formers (2). He found that these crystals were composed not of calcium oxalate, the most common solid phase found in patients with nephrolithiasis, but of calcium phosphate (3). He believed that the calcium phosphate crystals formed in the papillary interstitium and then eroded into the urinary space, serving as a heterogeneous nucleation surface for calcium oxalate. B. Finlayson later argued that, due to rapid flow of the renal ultrafiltrate through the tubule, there was insufficient time for formation of a lumenobstructing solid phase (4), which also suggested that an intratubular site of stone formation was unlikely. However, other investigators found that calcium oxalate crystals adhered to cultured tubular cells (5), where they could either be endocytosed or remain on the cell surface, serving as a nidus for growth into larger, clinically significant, calculi.

\section{Site of the initial solid phase}

Where is the site of initial crystallization - the interstitium, the tubular lumen, or perhaps the renal calyx, where supersaturated fluid awaits excretion into the ureter? Knowing the site of initial crystallization would improve understanding of the patho- genesis of stone formation and allow investigators to propose and test more focused hypotheses. This would help them to devise effective therapy aimed at preventing recurrent nephrolithiasis, which afflicts approximately 50\% of stone formers within five years of the initial stone (6). Yet until the elegant study by A.P. Evan et al. reported in this issue of the JCI (7), we did not have an answer to this rather elementary question. These investigators performed kidney biopsies on stoneforming patients to determine the anatomical site and composition of the initial solid phase. They sampled areas adjacent to Randall's plaques in patients undergoing percutaneous nephrolithotomy. In hypercalciuric calcium oxalate stone formers, they found initial calcium phosphate (apatite) crystallization in the basement membrane of the thin limbs of the loop of Henle (Figure 1) with subsequent extension to the vasa recta, then to the interstitial tissue surrounding the terminal collecting ducts, and finally, in the most severe cases, to the papillae. Erosion of this solid phase into the urinary space, which is supersaturated with respect to calcium oxalate, may have promoted heterogeneous nucleation and formation of kidney stones. In patients with hyperoxaluria resulting from intestinal bypass, the initial crystals were again a calcium phosphate complex, but these arose within the tubule lumens of terminal collecting ducts (Figure 2). Contact of these crystals with urine, supersaturated with respect to calcium oxalate, may have promoted heterogeneous nucleation and formation of kidney stones. Nonstone formers, subjected to nephrectomy, had neither plaque nor crystals. Thus there are different sites of initial 


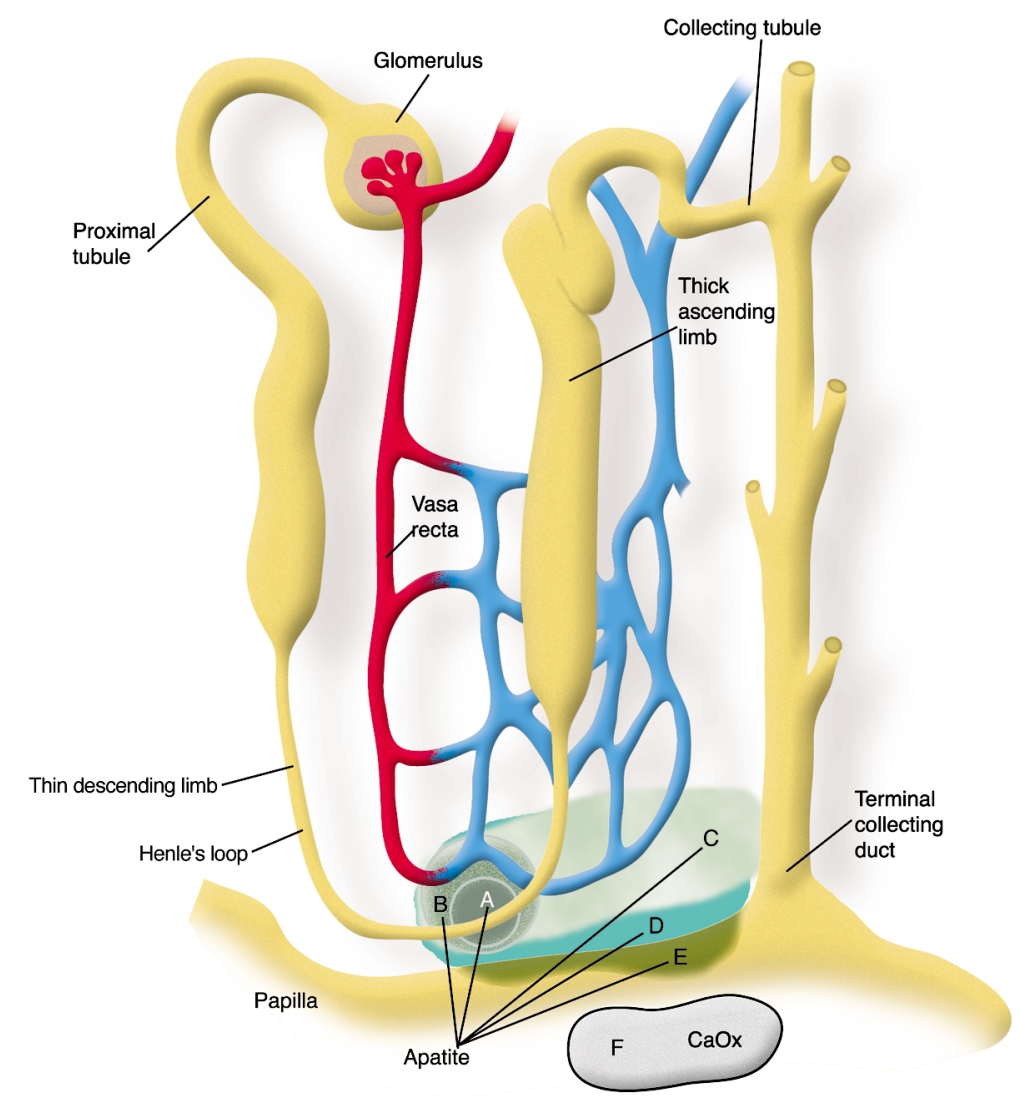

by Ken Beauchamp J. Clin. Invest.

\section{Figure 1}

Stone formation in patients with idiopathic hypercalciuria. Initial calcium phosphate (apatite) crystallization was found in the basement membrane of the thin limbs of the loop of Henle $(A)$ with subsequent extension to the vasa recta $(B)$, then to the interstitial tissue surrounding the terminal (inner medullary) collecting ducts $(C)$, and finally, in the most severe cases, extension to the papillae (D). Erosion of this solid phase into the urinary space $(E)$, which is supersaturated with respect to calcium oxalate $(\mathrm{CaOx})$, may have promoted heterogeneous nucleation and formation of calcium oxalate kidney stones $(F)$.

crystallization depending upon the metabolic abnormality leading to stone formation.

\section{Potential mechanisms for stone formation}

Why does the initial solid phase form in these distinct locations, and why are the initial crystals apparently only calcium phosphate? The basement membrane of the thin limb appears an unlikely site for initial crystallization in patients with idiopathic hypercalciuria. It is not the site of either vectorial calcium or phosphorus transport (8) and, since even the transtubular permeabilities of these ions are very low (8), it is difficult to link supersaturation within the thin limbs (9) to the surrounding interstitium. However, anatomically, the thin limbs are in very close proximity to the vasa recta and the collecting ducts, and all are situated in a highly concentrated, hypertonic environment. One could propose a sequence of events which might lead to increased supersaturation and subsequent crystal formation. Following ingestion and absorption of dietary calcium, the renalfiltered load of calcium would increase, resulting in increased tubular calcium concentration (10). The medullary countercurrent mechanism would concentrate the calcium extracted from the thick ascending limb into the hypertonic papilla. The vasa recta, also with an increased calcium concentration, would fail to readily remove calcium from the interstitium. The increased serum calcium would stimulate the calcium receptor and decrease reabsorption of water in the collecting duct (11), further concen- trating the interstitium. Vectorial proton transport into the collecting duct would alkalinize the interstitium. The $\mathrm{pH}$ of the vasa recta would also increase following gastric proton secretion, the so-called alkaline tide, resulting in less bicarbonate removal from the medullary interstitium. The increased $\mathrm{pH}$ would decrease the solubility of calcium phosphate complexes. Perhaps an extracellular matrix protein, specific to the papillary interstitium, could provide a site promoting heterogeneous nucleation (12), which occurs with a lower degree of supersaturation than homogeneous nucleation. Future studies will be necessary to test these hypotheses.

Intraluminal crystal formation in the collecting duct appears a more likely site for initial crystallization in patients following intestinal bypass surgery. The collecting duct fluid can be hypertonic with elevated concentrations of calcium leading to supersaturation. Yet the urine from the patients in Evan's study was undersaturated with respect to calcium phosphate, indicating that, thermodynamically, a stone should not form. However, the lack of demonstrable supersaturation may be a function of the 24-hour urine collection; the maximal supersaturation, and thus the propensity for stone formation, is never detected. While a 24-hour urine collection is an important predictor of the likelihood of forming stones, it is not the sole predictor. It seems probable that supersaturation initiates crystal formation, but we still do not understand the relationship between the degree of urinary supersaturation and stone disease.

\section{Future directions}

Now that we know where the initial solid phase forms, what are the next questions? Investigators studying the kidney generally concentrate on the effects of transport on tubular fluid ion concentration; the current study will force us to look more carefully at the effects of basolateral membrane transport on interstitial ion concentrations. We know little about supersaturation in this critical region of the kidney, yet this is where the majority of stones originate.

Unneeded calcium and oxalate must be excreted in a minimal amount of urine to rid the body of these potential 


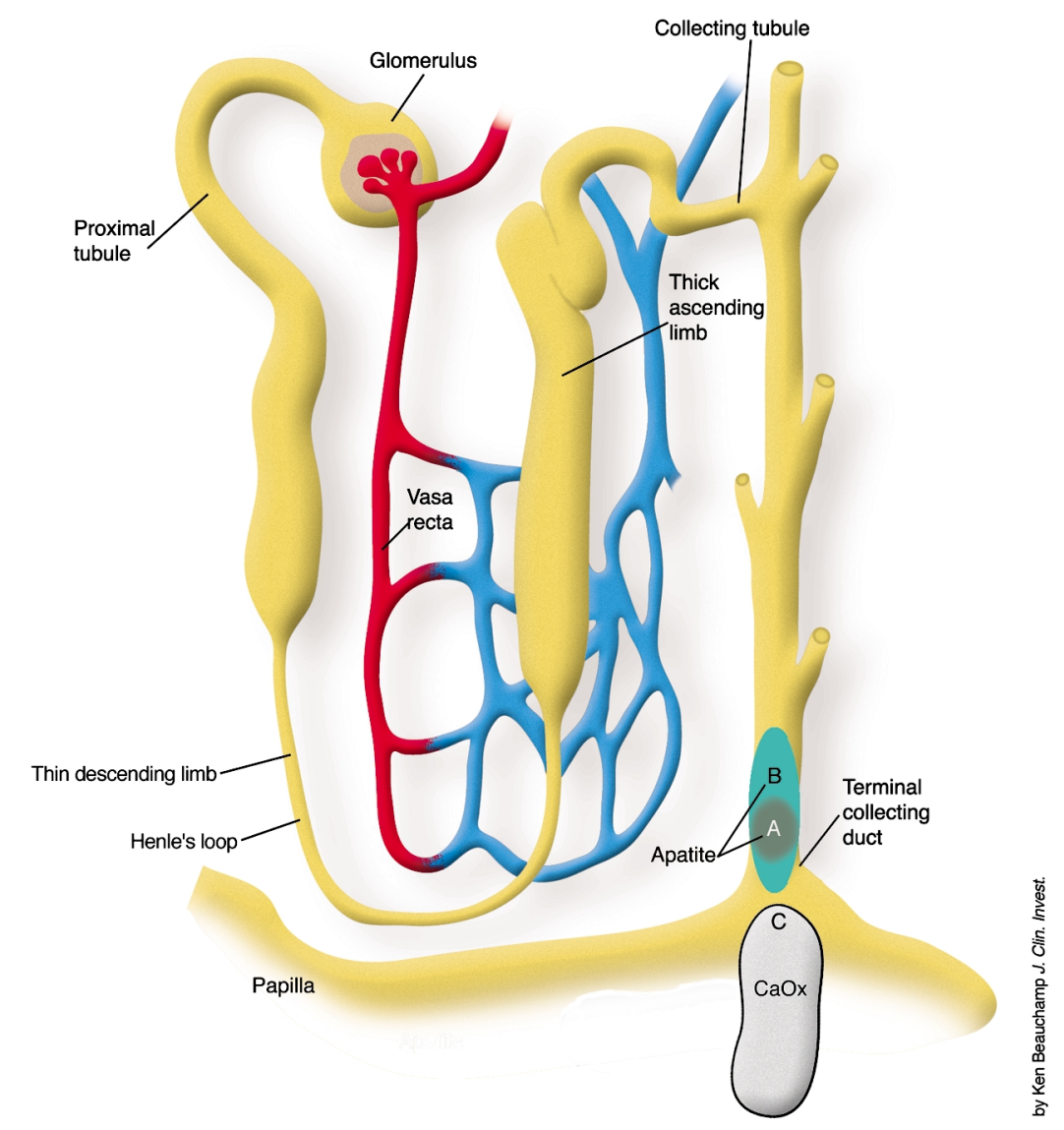

Figure 2

Stone formation in patients following intestinal bypass. Initial calcium phosphate (apatite) crystallization was found within the tubule lumens of the terminal collecting ducts ( $A$ and $B$ ). Contact with the urine, supersaturated with respect to calcium oxalate, may have promoted heterogeneous nucleation and formation of calcium oxalate kidney stones (C).

toxins while conserving extracellular fluid volume. Human urine and blood are supersaturated with respect to calcium oxalate (1) and calcium hydrogen phosphate (13), respectively, yet solid phases rarely form because we produce inhibitors to initial nucleation and subsequent aggregation (12). In rats, stone formation occurs when the magnitude of the supersaturation overcomes this potent inhibition (14). The current study should point us in the direction of investigating the relationship between supersaturation and inhibitor proteins not only in the urine but in the interstitium as well.

Crystals may stimulate production of proteins, such as osteopontin, which appear to regulate growth of the solid phase (15). In patients with idiopathic hypercalciuria, there was evidence for crystal-induced cell injury in areas of dense crystal deposition, while in the bypass patients there was not only cell injury but also cell death (7). Were levels of osteopontin increased in the medullary interstitium in either of these types of stone formers?

\section{An animal model of stone formation}

The genetic hypercalciuric stoneforming rat exhibits metabolic abnormalities similar to patients with idiopathic hypercalciuria in that these rats absorb excessive amounts of intestinal calcium, they fail to adequately reabsorb filtered calcium, and their bone resorption is uniquely sensitive to $1,25(\mathrm{OH})_{2} \mathrm{D}_{3}$. All of these characteristics are apparently due to an increase in the number of receptors for vitamin $\mathrm{D}(16,17)$. The genetic hypercalciuric stone-forming rats spontaneously form calcium phosphate stones (18) similar to those found in this study unless their diet is augmented with an oxalate precursor (19). Both hypercalciuric rats and humans appear to be predisposed to initially form calcium phosphate stones and not the commonly observed calcium oxalate stones. In both rats (20) and humans (21) the upper limit of metastability, that level of supersaturation at which a solid phase forms, increases with increasing calcium oxalate, but not calcium phosphate, supersaturation. Thus rats and humans appear protected against calcium oxalate stone formation unless a nucleation site, such as the more easily formed calcium phosphate crystal, is present.

This study highlights the role of physician scientists working with basic scientists in medical research to jointly address important problems using sophisticated clinical and laboratory techniques and then applying these results to refine hypotheses for further testing. Agile movement between the bedside and the bench, as exemplified in this study, will provide insight into, and ultimately prevention of, disorders such as nephrolithiasis.

\section{Acknowledgments}

This work was supported in part by NIH Grants AR 46289, DK 57716, and DK 56788.

1. Monk, R.D., and Bushinsky, D.A. 2003. Kidney stones. In Williams textbook of endocrinology. P.R Larsen, H.M. Kronenberg, S. Melmed, and K.S Polonsky, editors. W.B.Saunders. Philadelphia, Pennsylvania, USA. 1411-1425.

2. Randall, A. 1937. The origin and growth of renal calculi. Ann. Surg. 105:1009-1027.

3. Randall, A. 1940. Papillary pathology as a precursor of primary renal calculus. J. Urol. 44:580-589.

4. Finlayson, B., and Reid, F. 1978. The expectation of free and fixed particles in urinary stone disease. Invest. Urol. 15:442-448.

5. Lieske, J.C., and Toback, F.G. 2000. Renal cell-urinary crystal interactions. Curr. Opin. Nephrol. Hypertens. 9:349-355.

6. Asplin, J.R., Favus, M.J., and Coe, F.L. 2000 Nephrolithiasis. In The kidney. B.M. Brenner, editor. W.B. Saunders Company. Philadelphia, Pennsylvania, USA. 1774-1819.

7. Evan, A.P., et al. 2003. Randall's plaque of patients with nephrolithiasis begins in basement membranes of thin loops of Henle. J. Clin. Invest. 111:607-616. doi:10.1172/JCI200317038.

8. Rocha, A.S., Magaldi, J.B., and Kokko, J.P. 1977. Calcium and phosphate transport in isolated segments of rabbit Henle's loop. J. Clin. Invest. 59:975-983.

9. Asplin, J.R., Mandel, N.S., and Coe, F.L. 1996. Evidence for calcium phosphate supersaturation in the loop of Henle. Am. J. Physiol. 270:F604-F613.

10. Bushinsky, D.A., and Monk, R.D. 1998. Calcium. Lancet. 352:306-311.

11. Hebert, S.C., Brown, E.M., and Harris, H.W. 1997. Role of the $\mathrm{Ca}^{2+}$-sensing receptor in divalent min eral ion homeostasis. J. Exp. Biol. 200:295-302.

12. Coe, F.L., and Parks, J.H. 1997. New insights into the pathophysiology and treatment of nephrolithiasis: new research venues. J. Bone. 
Miner. Res. 12:522-533.

13. Neuman, W.F., and Neuman, M.W. 1958. The chemical dynamics of bone mineral. University of Chicago Press. Chicago, Illinois, USA. 209 pp.

14. Bushinsky, D.A., Parker, W.R., and Asplin, J.R. 2000. Calcium phosphate supersaturation regulates stone formation in genetic hypercalciuric stone-forming rats. Kidney Int. 57:550-560.

15. Xie, Y., et al. 2001. Expression, roles, receptors, and regulation of osteopontin in the kidney. Kidney Int. 60:1645-1657.
16. Bushinsky, D.A. 1999. Genetic hypercalciuric stone-forming rats. Curr. Opin. Nephrol. Hypertens. 8:479-488.

17. Li, X.-Q., Tembe, V., Horwitz, G.M., Bushinsky, D.A., and Favus, M.J. 1993. Increased intestinal vitamin $\mathrm{D}$ receptor in genetic hypercalciuric rats: a cause of intestinal calcium hyperabsorption. J. Clin. Invest. 91:661-667.

18. Bushinsky, D.A., Grynpas, M.D., Nilsson, E.L., Nakagawa, Y., and Coe, F.L. 1995. Stone formation in genetic hypercalciuric rats. Kidney Int.
48:1705-1713.

19. Bushinsky, D.A., et al. 2002. Calcium oxalate stone formation in genetic hypercalciuric stoneforming rats. Kidney Int. 61:975-987.

20. Asplin, J.R., et al. 1997. Relationship between supersaturation and crystal inhibition in hypercalciuric rats. Kidney Int. 51:640-645.

21. Asplin, J.R., Parks, J.H., and Coe, F.L. 1997. Dependence of upper limit of metastablility on supersaturation in nephrolithiasis. Kidney Int 52:1602-1608. 